\title{
The amyloid- $\beta$ isoform pattern in cerebrospinal fluid in familial PSEN1 M139T- and L286P-associated Alzheimer's disease
}

\author{
ERIK PORTELIUS $^{1}$, JUAN FORTEA ${ }^{2}$, JOSE LUIS MOLINUEVO ${ }^{2}$, MIKAEL K. GUSTAVSSON ${ }^{1}$, \\ ULF ANDREASSON $^{1}$ and RAQUEL SANCHEZ-VALLE ${ }^{2}$
}

\author{
${ }^{1}$ Department of Psychiatry and Neurochemistry, Institute of Neuroscience and Physiology, The Sahlgrenska Academy \\ at the University of Gothenburg, Mölndal, Sweden; ${ }^{2}$ Genetic Counseling Program for Familial Dementias, \\ Alzheimer's Disease and Other Cognitive Disorders Unit, Neurology Department, \\ Hospital Clinic, IDIBAPS, Barcelona, Spain
}

Received October 13, 2011; Accepted January 12, 2012

DOI: $10.3892 / \mathrm{mmr} .2012 .774$

\begin{abstract}
There are several familial forms of Alzheimer's disease (AD) most of which are caused by mutations in the genes that encode the presenilin enzymes involved in the production of amyloid- $\beta(A \beta)$ from the amyloid precursor protein (APP). In $\mathrm{AD}, \mathrm{A} \beta$ forms fibrils that are deposited in the brain as plaques. Much of the fibrillar $A \beta$ found in the plaques consists of the 42 amino acid form of $A \beta(A \beta 1-42)$ and it is now widely accepted that $A \beta$ is related to the pathogenesis of $A D$ and that $A \beta$ may both impair memory and be neurotoxic. In human cerebrospinal fluid (CSF) several C-and N-terminally truncated $\mathrm{A} \beta$ isoforms have been detected and their relative abundance pattern is thought to reflect the production and clearance of $\mathrm{A} \beta$. By using immunoprecipitation and mass spectrometry, we have previously demonstrated that carriers of the familial AD (FAD)-associated PSEN1 A431E mutation have low CSF levels of C-terminally truncated $A \beta$ isoforms shorter than A $\beta 1-40$. Here we replicate this finding in symptomatic carriers of the FAD-causing PSEN1 L286P mutation. Furthermore, we show that preclinical carriers of the PSENI M139T mutation may overexpress A $\beta 1-42$ suggesting that this particular mutation may cause AD by stimulating $\gamma$-secretasemediated cleavage at amino acid 42 in the $A \beta$ sequence.
\end{abstract}

\section{Introduction}

According to the amyloid cascade hypothesis, there is an imbalance between the production and clearance of amyloid- $\beta(\mathrm{A} \beta)$,

Correspondence to: Dr Erik Portelius, Clinical Neurochemistry Laboratory, Institute of Neuroscience and Physiology, Department of Psychiatry and Neurochemistry, The Sahlgrenska Academy at the University of Gothenburg, Sahlgrenska University Hospital, Mölndal, SE-431 80 Mölndal, Sweden E-mail: erik.portelius@neuro.gu.se

Key words: familial Alzheimer's, mass spectrometry, presenilin, amyloid- $\beta$ isoforms leading to the formation of insoluble aggregates and soluble monomers and larger assemblies of $A \beta$ which drive the synaptic loss and neuronal degeneration in the Alzheimer's disease (AD) process (1). Data supporting the view that life-long amyloid precursor protein (APP) overexpression triggers $A \beta$ deposition and neurodegeneration come from studies on Down's syndrome patients and the finding of a duplication of the APP locus in a certain familial form of $\operatorname{AD}(2,3)$.

$A \beta$ is produced through the amyloidogenic pathway from APP by proteolytic cleavage involving the two aspartyl proteases $\beta$ - and $\gamma$-secretase generating $A \beta 1-42$ and $C$-terminal truncated $A \beta$ isoforms ranging from $A \beta 1-40$ down to A $\beta 1-17(4,5)$. In another catabolic APP pathway, $\beta$-secretase cleavage in conjunction with $\alpha$-secretase cleavage result in the release of several short $A \beta$ isoforms (A $\beta 1-16$ down to $A \beta 1-13$ ) (5) of which A 11-16 previously have been shown to be elevated in sporadic $\mathrm{AD}$ (SAD) (6) and familial AD (FAD) (7) compared to non-demented controls. Moreover, this pathway is induced upon pharmacological $\gamma$-secretase inhibitor treatment of cells, mice, dogs, rhesus monkeys and humans (5,8-11).

The first identified mutation causing FAD was a missense mutation in the APP gene (12). However, most FAD are caused by mutations in the highly homologous genes of the presenilin enzymes that constitute the active site $\gamma$-secretase, presenilin 1 (PSEN1) and presenilin 2 (PSEN2) $(13,14)$. These mutations appear to accelerate $A \beta$ plaque formation and have been shown to increase the $A \beta 1-42 / A \beta 1-40$ ratio in primary fibroblasts and plasma of affected individuals (15-18). Using immunoprecipitation in combination with mass spectrometry, we recently showed that carriers of the FAD-associated PSENI A431E mutation have low CSF levels of $A \beta 1-37, A \beta 1-38$ and $A \beta 1-39$ (7). Here we investigated the $A \beta$ isoform pattern in the CSF from carriers of the FAD-associated PSEN1 M139T and L286P mutations.

\section{Materials and methods}

Study participants. Participants were recruited from the Genetic Counseling Program for Familial Dementias (PICOGEN) at the Hospital Clinic, Barcelona, Spain (19). Eleven subjects from 3 families with 2 PSEN1 mutations 
Table I. Summary of the 11 subjects included in the study.

\begin{tabular}{|c|c|c|c|c|c|c|}
\hline Subject no. & Carrier & Mutation & CDRsum & CDRtot & MMSE & Adjusted age \\
\hline 1 & 1 & L286P & 2 & 0.5 & 28 & -2.7 \\
\hline 2 & 1 & L286P & 2.5 & 0.5 & 24 & 2.6 \\
\hline 3 & 1 & L286P & 5.5 & 1 & 24 & 4.7 \\
\hline 4 & 0 & L286P & 0 & 0 & 30 & -5.2 \\
\hline 5 & 0 & L286P & 0 & 0 & 29 & -1.2 \\
\hline 6 & 0 & L286P & 0 & 0 & 29 & 3.8 \\
\hline 7 & 1 & M139T & 0 & 0 & 30 & -21.8 \\
\hline 8 & 1 & M139T & 0 & 0 & 30 & -13.1 \\
\hline 9 & 1 & M139T & 0 & 0 & 28 & -12.5 \\
\hline 10 & 0 & M139T & 0 & 0 & 29 & -20.9 \\
\hline 11 & 0 & M139T & 0 & 0 & 29 & -10.6 \\
\hline
\end{tabular}

CDRsum, clinical dementia rating scale sum of boxes; CDRtot, clinical dementia rating scale total score; MMSE, mini-mental state examination score.

(L286P, M139T) (6 mutation carriers and 5 non-carriers) were included in the study $(20,21)$. The L286P mutation causes familial early onset $\mathrm{AD}$ at a median age of 40 years and cerebral hematomas in $42 \%$ of the affected subjects. The neuropathological studies in this family reveal the typical features of $\mathrm{AD}$ associated with severe amyloid angiopathy and 'cotton-wool plaques'. The M139T mutation causes familial early onset AD at a median age of 46 with typical clinical and neuropathological characteristics of AD associated with intense amyloid angiopathy but no 'cotton-wool' plaques or lobar hematomas. Because of the relatively predictable age of onset within a family we calculate for each participant the 'adjusted age' as the subject's age relative to the median familial age of onset. Subjects underwent clinical and cognitive evaluation and were classified clinically as asymptomatic if they had normal cognitive evaluation and the Clinical Dementia Rating (CDR) scale was equal to 0; or symptomatic if cognitive performance was more than 1.5 SD below the mean, with respect to age and education level, in any in any cognitive test or CDR $>0$ (Table I).

CSF analysis. Ten milligrams ofCSF was obtained in the morning using a 22 gauge Sprotte needle. The CSF was then centrifuged, aliquoted into siliconized polypropylene Eppendorf tubes and frozen at $-80^{\circ} \mathrm{C}$ within $2 \mathrm{~h}$ of being obtained.

The study was approved by the Hospital Clinic Ethics Committee, and all participants gave informed consent to participate in the study, which was conducted according to the provisions of the Helsinki Declaration.

Immunoprecipitation (IP)-mass spectrometry (MS). IP and MS analyses were conducted as previously described with the monoclonal antibody 6E10 (epitope 4-9, Signet Laboratories Inc., Dedham, MA, USA) and matrix-assisted laser desorption/ ionization time-of-flight mass spectrometry (MALDI-TOFMS, Autoflex, Bruker Daltonics, Bremen, Germany) operating in reflector mode (22). The peak areas were normalized to the sum of the integrated peaks which result in a relative abundance rather than an absolute concentration pattern (6).

\section{Results}

Representative CSF A $\beta$ isoform mass spectra from a PSEN1 L286P FAD mutation carrier and a non-carrier are shown in Fig. 1. All isoforms $(A \beta 1-13, A \beta 1-14, A \beta 1-15, A \beta 1-16, A \beta 1-17$, $\mathrm{A} \beta 1-18, \mathrm{~A} \beta 1-19, \mathrm{~A} \beta 1-20, \mathrm{~A} \beta 1-30, \mathrm{~A} \beta 1-33, \mathrm{~A} \beta 1-34, \mathrm{~A} \beta 1-37$, $\mathrm{A} \beta 1-38, A \beta 1-39, \mathrm{~A} \beta 1-40$ and $\mathrm{A} \beta 1-42)$ were reproducibly detected from all subjects and quantified in respect to their relative abundance.

The MS peaks at mass-to-charge ratio 4073.0, 4130.0, 4229.1 and 4512.2, corresponding to $\mathrm{A} \beta 1-37, \mathrm{~A} \beta 1-38, \mathrm{~A} \beta 1-39$ and $\mathrm{A} \beta 1-42$, respectively, were lower in the PSEN1 L286P FAD mutation carriers (Fig. 2). The relative level of A $\beta 1-37$ was reduced by $31 \%, A \beta 1-38$ by $21 \%, A \beta 1-39$ by $29 \%$ and $A \beta 1-42$ by $81 \%$. In contrast $A \beta 1-15$ and $A \beta 1-20$ were increased by 47 and $34 \%$, respectively, while the other isoforms detected were more or less unaffected.

In contrast, PSEN1 M139T carriers displayed similar levels as non-carriers of all $\mathrm{A} \beta$ isoforms reproducibly detected except $A \beta 1-42$ that was present at $41 \%$ higher levels in the group harboring the mutation as compared to noncarriers (Fig. 2).

\section{Discussion}

The reduction in $\mathrm{A} \beta 1-42$ in the CSF from AD patients is a well-replicated finding thought to reflect the AD pathology with plaques in the brain acting as sinks trapping A $\beta 1-42$ (23). The data presented here on L286P mutation carriers suggests that this specific PSEN1 mutation modulates $\gamma$-secretase function, which is reflected by decreased levels of $A \beta 1-37, A \beta 1-38$ and $A \beta 1-39$, in a similar manner as the PSEN1 A431E mutation. It has been speculated that the decrease in A $\beta 1-37, \mathrm{~A} \beta 1-38, \mathrm{~A} \beta 1-39$, due to the PSEN1 A431E mutation, indicates that the $\gamma$-secretase cleavage site preference has been changed in a disease-promoting manner (7). Similar changes in the levels of the isoforms have also been reported from cells expressing the PSEN1 $\triangle 9$ or L166P mutation, or the PSEN2 N141I mutation (15). Thus, 


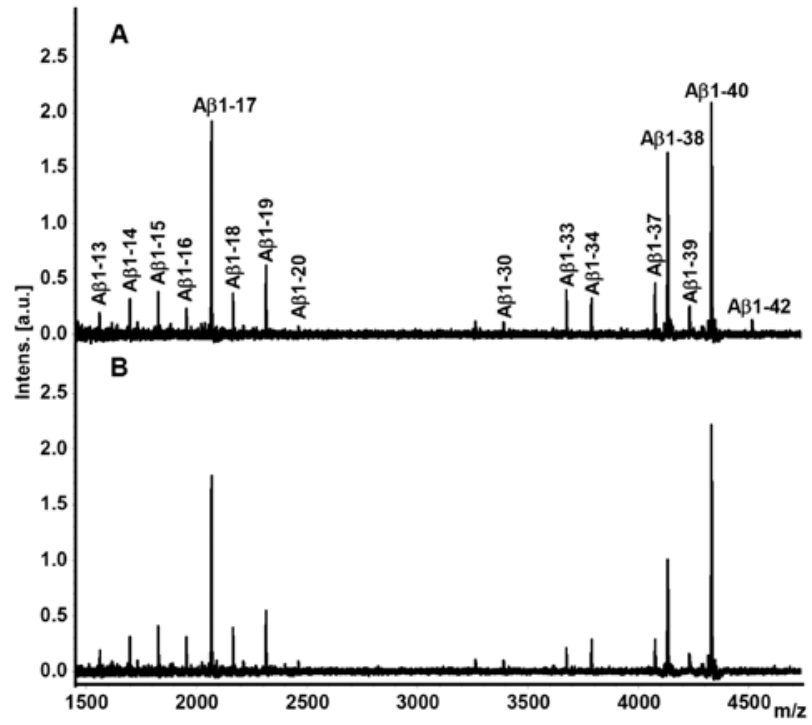

Figure 1. Representative MALDI-TOF spectra showing A $\beta$ isoforms immunoprecipitated from CSF using the antibody $6 \mathrm{E} 10$. (A) Representative $A \beta$ isoform pattern in a control individual. (B) Representative A $\beta$ isoform pattern in a carrier of the FAD-associated PSEN1 L286P mutation.
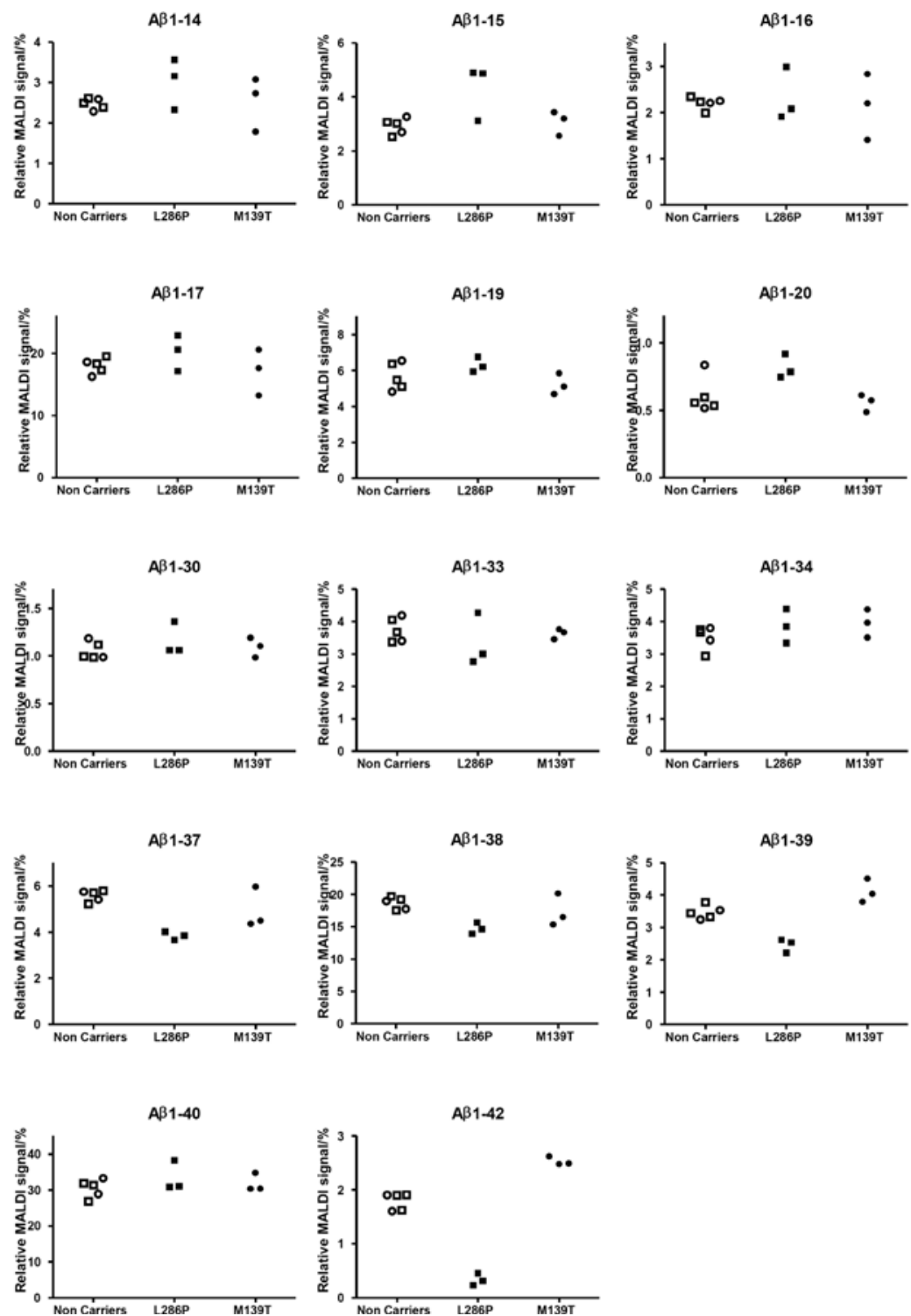

Figure 2. Scatter plots displaying the distribution of the A $\beta$ isoform pattern in carriers (filled squares) and non-carriers (open squares) of the FAD-associated PSEN1 L286P mutation and carriers (filled circles) and non-carriers (open circles) of the FAD-associated PSEN1 M139T mutation. 
modulating the $\gamma$-secretase function to boost cleavages at Gly37, Gly38 and Val39 may be an attractive approach to prevent aggregation of $A \beta$ and consequently the formation of plaques.

The increase in A $\beta 1-20$ in L286P mutation carriers is a finding which previously has been described for the PSEN1 A431E mutation (7). Two candidate enzymes which possibly can cleave at this position are insulin-degrading enzyme and BACE 2 (24). However, the underlying molecular mechanism needs to be further investigated.

We could not detect an increase in A $\beta 1-16$ for L286P mutation carriers as previously described for both sporadic and familial AD patients $(6,7)$. On the other hand, an increase by $47 \%$ was observed for A $\beta 1-15$. Whether this is a specific finding for this particular mutation needs to be confirmed in a larger patient sample.

The median age at onset for the PSEN1 L286P mutation is 40 years. The patients included in this group had a mean age of 41.5 thus very close to the critical age and they all displayed cognitive decline. In contrast, the mean age in the patient group carrying the PSEN1 M139T mutation was 30.2 years, which is almost 16 years younger than the expected age of onset (Table I). Furthermore, L286P seems to be a more aggressive mutation than M139T with cotton-wool plaques noted in the neuropath vs. no cotton-wool present in the M139T mutation. The A431E mutation also presents a median age of onset of 40 and cotton-wool plaques, suggesting similarities in the biological effects of L286P and A431E different from M139T.

The slightly increased relative levels of $A \beta 1-42$ in the CSF of M139T mutation carriers suggest that this particular mutation starts with an over-production of $A \beta$ very early in life or that these subjects present normal values of A $\beta 1-42$ while L286P mutation carriers present very low levels. Furthermore, the patients harboring the PSEN1 M139T mutation were all cognitively healthy (Table I).

It should be noted that due to the low number of individuals in the groups analyzed, no statistical analysis was performed. Moreover, there are also several non-quantitative aspects of IP-MS, which should be considered, as discussed elsewhere (25). However, the results presented here with decreased CSF levels of A $\beta 1-37, A \beta 1-38$ and $A \beta 1-39$ in patients with a PSEN1 L286P mutation are in agreement with previous findings in PSEN1 A431E mutation carriers and suggest that the pathogenic effect of some PSEN1 mutations may be related to impaired ability of the $\gamma$-secretase complex to produce C-terminally truncated $A \beta$ peptides that may inhibit $A \beta 1-42$ oligomerization.

In conclusion, the data suggest that IP-MS analysis of the CSF A $\beta$ isoform pattern may function as an additional screening tool to detect certain forms of PSEN1 mutations in patients with a family history indicating FAD. This hypothesis will be further tested on subjects having other PSEN1 mutations.

\section{Acknowledgements}

This study was supported by grants from the Swedish Research Council (projects 2006-6227, 2006-2740 and 2006-3505), the Alzheimer's Association (NIRG-08-90356), The Torsten and Ragnar Söderberg Foundation, Demensförbundet, cNEUPRO, the Royal Swedish Academy of Sciences, the
Sahlgrenska University Hospital, the Inga-Britt and Arne Lundberg Research Foundation, the Göteborg Medical Society, the Swedish Medical Society, Swedish Brain Power, Stiftelsen Gamla Tjänarinnor, Gun och Bertil Stohnes stiftelse, Åhlén-stiftelsen, Alzheimer Foundation, Sweden and the Spanish Ministry of Science and Technology (FIS080036).

\section{References}

1. Hardy JA and Higgins GA: Alzheimer's disease: the amyloid cascade hypothesis. Science 256: 184-185, 1992.

2. Lemere CA, Blusztajn JK, Yamaguchi H, Wisniewski T, Saido TC and Selkoe DJ: Sequence of deposition of heterogeneous amyloid beta-peptides and APO E in Down syndrome: implications for initial events in amyloid plaque formation. Neurobiol Dis 3: 16-32, 1996.

3. Rovelet-Lecrux A, Hannequin D, Raux G, et al: APP locus duplication causes autosomal dominant early-onset Alzheimer disease with cerebral amyloid angiopathy. Nat Genet 38: 24-26, 2006.

4. Beher D, Wrigley JD, Owens AP and Shearman MS: Generation of C-terminally truncated amyloid-beta peptides is dependent on gamma-secretase activity. J Neurochem 82: 563-575, 2002.

5. Portelius E, Price E, Brinkmalm G, et al: A novel pathway for amyloid precursor protein processing. Neurobiol Aging 32: 1090-1098, 2011.

6. Portelius E, Zetterberg H, Andreasson U, et al: An Alzheimer's disease-specific beta-amyloid fragment signature in cerebrospinal fluid. Neurosci Lett 409: 215-219, 2006.

7. Portelius E, Andreasson U, Ringman JM, et al: Distinct cerebrospinal fluid amyloid beta peptide signatures in sporadic and PSEN1 A431E-associated familial Alzheimer's disease. Mol Neurodegener 5: 2, 2010.

8. Portelius E, Van Broeck B, Andreasson U, et al: Acute effect on the Abeta isoform pattern in CSF in response to $\gamma$-secretase modulator and inhibitor treatment in dogs. J Alzheimers Dis 21: 1005-1012, 2010

9. Portelius E, Zhang B, Gustavsson MK, et al: Effects of gammasecretase inhibition on the amyloid beta isoform pattern in a mouse model of Alzheimer's disease. Neurodegener Dis 6: 258-262, 2009.

10. Portelius E, Dean RA, Gustavsson MK, et al: A novel Abeta isoform pattern in CSF reflects gamma-secretase inhibition in Alzheimer disease. Alzheimers Res Ther 2: 7, 2010.

11. Cook JJ, Wildsmith KR, Gilberto DB, et al: Acute gammasecretase inhibition of nonhuman primate CNS shifts amyloid precursor protein (APP) metabolism from amyloid-beta production to alternative APP fragments without amyloid-beta rebound. J Neurosci 30: 6743-6750, 2010.

12. Goate A, Chartier-Harlin MC, Mullan M, et al: Segregation of a missense mutation in the amyloid precursor protein gene with familial Alzheimer's disease. Nature 349: 704-706, 1991.

13. Levy-Lahad E, Wasco W, Poorkaj P, et al: Candidate gene for the chromosome 1 familial Alzheimer's disease locus. Science 269: 973-977, 1995.

14. Sherrington R, Rogaev EI, Liang Y, et al: Cloning of a gene bearing missense mutations in early-onset familial Alzheimer's disease. Nature 375: 754-760, 1995.

15. Bentahir M, Nyabi O, Verhamme J, et al: Presenilin clinical mutations can affect gamma-secretase activity by different mechanisms. J Neurochem 96: 732-742, 2006.

16. Citron M, Westaway D, Xia W, et al: Mutant presenilins of Alzheimer's disease increase production of 42-residue amyloid beta-protein in both transfected cells and transgenic mice. Nat Med 3: 67-72, 1997.

17. Kumar-Singh $\mathrm{S}$, Theuns $\mathrm{J}$, Van Broeck $\mathrm{B}$, et al: Mean ageof-onset of familial alzheimer disease caused by presenilin mutations correlates with both increased Abeta42 and decreased Abeta40. Hum Mutat 27: 686-695, 2006.

18. Ringman JM, Younkin SG, Pratico D, et al: Biochemical markers in persons with preclinical familial Alzheimer disease. Neurology 71: 85-92, 2008.

19. Fortea J, Llado A, Clarimon J, et al: PICOGEN: Five years experience with a genetic counselling program for dementia. Neurologia 26: 143-149, 2011. 
20. Sanchez-Valle R, Llado A, Ezquerra M, Rey MJ, Rami L and Molinuevo JL: A novel mutation in the PSEN1 gene (L286P) associated with familial early-onset dementia of Alzheimer type and lobar haematomas. Eur J Neurol 14: 1409-1412, 2007.

21. Fortea J, Llado A, Bosch B, et al: Cerebrospinal fluid biomarkers in Alzheimer's disease families with PSEN1 mutations. Neurodegener Dis 8: 202-207, 2011.

22. Portelius E, Tran AJ, Andreasson U, et al: Characterization of amyloid beta peptides in cerebrospinal fluid by an automated immunoprecipitation procedure followed by mass spectrometry. J Proteome Res 6: 4433-4439, 2007.
23. Blennow K,Hampel H, Weiner M and Zetterberg H: Cerebrospinal fluid and plasma biomarkers in Alzheimer disease. Nat Rev Neurol 6: 131-144, 2010.

24. Andreasson U, Portelius E, Andersson ME, Blennow K and Zetterberg H: Aspects of beta-amyloid as a biomarker for Alzheimer's disease. Biomark Med 1: 59-78, 2007.

25. Portelius E, Bogdanovic N, Gustavsson MK, et al: Mass spectrometric characterization of brain amyloid beta isoform signatures in familial and sporadic Alzheimer's disease. Acta Neuropathol 120: 185-193, 2010. 